\title{
Apoptosis, Rather Than Oncosis, is the Predominant Mode of Spontaneous Death of Isolated Adult Rat Cardiac Myocytes in Culture
}

\author{
Satoshi Kato, MD; Genzou Takemura, MD; Rumi Maruyama, BS; \\ Takuma Aoyama, MD; Kenji Hayakawa, MD; Masahiko Koda, MD; \\ Yukinori Kawase, MD; Yiwen Li, MD; Shinya Minatoguchi, MD; \\ Takako Fujiwara, MD*; Hisayoshi Fujiwara, MD
}

\begin{abstract}
A number of isolated adult cardiomyocytes dies within a few days of culture and the mode of death has recently been suggested to be apoptosis, based on its association with the appearance of DNA fragmentation. However, morphological evidence is still lacking and precise analysis, including quantification, has not been performed. Adult rat ventricular cardiomyocytes isolated by enzymatic dissociation were incubated for 7 days in a serum-free medium (the rapid attachment model) and after various incubation periods, both attached and floating cells were counted and classified based on combined criteria of morphology and membrane permeability (dye exclusion): type 1, rod cells with intact membranes; type 2, non-rod cells with intact membranes; and type 3, non-rod cells with ruptured membranes. The number of both rod-shaped and dye-excluding cells decreased with the incubation period. After 7 days culture, the number of residual cells decreased to $12 \%$ of the initial value. Electron microscopy identified type 1 cells as viable, type 2 cells as viable or apoptotic, and type 3 as undergoing oncosis (primary necrosis) or secondary post-apoptotic necrosis. Ultrastructural morphometry revealed that oncotic cell death occurred predominantly during the early phase of culture whereas the more abundant apoptotic cell death occurred throughout the culture period. In conclusion, although both apoptotic and oncotic death occur in the natural course of adult rat cardiomyocytes in short-term culture, apoptosis is more predominant. Because of the high incidence of spontaneous cell death predominantly via apoptosis, this information is important for the interpretation of studies using this cell type in culture. (Jpn Circ J 2001; 65: 743-748)
\end{abstract}

Key Words: Apoptosis; Cardiac myocytes; Necrosis; Oncosis; Ultrastructure

$\mathbf{T}$ here are 2 models of cultured isolated adult mammalian cardiomyocytes as described by Jacobson and Piper: ${ }^{1}$ the redifferentiated model and the rapid attachment model. In the redifferentiated model, serum supplements the culture media and the cardiomyocytes undergo a gradual morphologic transition from a rod to a spheroid shape ${ }^{2-4}$ These cells contract spontaneously, but lose their specific structure as myocytes during the gradual transition. Although the cells partially regain myotypic structure during a subsequent flattening and spreading phase and maintain contraction for up to 60 days, there is a problem with the validity of this model for terminally differentiated cells in vivo! In contrast, the isolated adult cardiomyocytes in the rapid attachment model rapidly attach to culture dishes precoated with adhesive reagents and are then cultured in a serum-free medium?-7 The attached cells do not contract spontaneously, but maintain the characteristic morphology of intact cardiomyocytes. The most serious problem with this model is that the number of

(Received February 9, 2001; revised manuscript received April 3, 2001; accepted April 24, 2001)

Second Department of Internal Medicine, Gifu University School of Medicine, Gifu and *Department of Food Science, Kyoto Women's University, Kyoto, Japan

Mailing address: Hisayoshi Fujiwara, MD, PhD, Second Department of Internal Medicine, Gifu University School of Medicine, 40 TsukasaMachi, Gifu 500-8705, Japan. E-mail: gifuim-gif@umin.u-tokyo. ac.jp adherent cells decrease so rapidly via spontaneous death that their use for experiments is limited; ${ }^{1}$ most die within 1 week.

Yamamoto et al investigated the spontaneous death of isolated adult rat cardiomyocytes in culture, but they used the redifferentiated model, not the rapid attachment model in which the short life of the system is more problematic. Their study reported cardiomyocyte apoptosis on the basis of the presence of DNA fragmentation, a biochemical hallmark of apoptosis,, 10 without evidence of the characteristic ultrastructure that is the morphological hallmark and gold standard for apoptosis at present ${ }^{11-13}$ Accurate information on the natural course is important in such short living cells as adult ventricular cardiomyocytes because spontaneous death may have a significant influence on the interpretation of experimental results, particularly in studies regarding cell kinetics. In the present study, therefore, we tried to delineate the spontaneous death of isolated adult rat ventricular cardiomyocytes in the rapid attachment model using both morphological and biochemical hallmarks of apoptosis and oncosis.

\section{Methods}

Isolation and Culture of Cardiomyocytes

The isolation and culture of adult rat ventricular cardiomyocytes were performed using previously established techniques with slight modifications, 5,6,14 Adult male 
Sprague-Dawley rats (200-250g) were injected with 500 units $/ \mathrm{kg}$ heparin sodium intraperitoneally and then anesthetized with intraperitoneal pentobarbital $(30 \mathrm{mg} / \mathrm{kg})$. The heart was removed and hooked up to a modified Langendorff perfusion apparatus (Kontes) and washed for $5 \mathrm{~min}$ with $\mathrm{Ca}^{2+}$-free modified Krebs-Ringer buffer containing $(\mathrm{mmol} / \mathrm{L})$ $\mathrm{NaCl} 110, \mathrm{KCl} 2.6, \mathrm{MgSO}_{4} 1.2, \mathrm{KH}_{2} \mathrm{PO} 41.2, \mathrm{NaHCO}_{3}$ 25.0, HEPES 25.0, and glucose 11.0. The heart was then recirculated with buffer containing $0.1 \%$ collagenase type II (Worthington Biochemical Co) and $0.1 \%$ albumin. Fifteen minutes was allowed for $\mathrm{Ca}^{2+}$-free circulation, and then $\mathrm{Ca}^{2+}$ was added gradually to a maximal concentration of 1 $\mathrm{mmol} / \mathrm{L}$ over a $45-\mathrm{min}$ period. At the end of the perfusion, both ventricles were removed, cut into small pieces, and incubated in fresh buffer containing $0.1 \%$ collagenase and $1 \%$ albumin for $10-20$ min while being gently rotated. The suspension was filtered through a $209 \mu \mathrm{m}$ nylon mesh and centrifuged for $5 \mathrm{~min}$ at $50 \mathrm{G}$. The cell pellet was washed with $0.2 \%$ albumin and centrifuged for $5 \mathrm{~min}$ at $50 \mathrm{G}$ through a density gradient of $0.2 \%$ and $4 \%$ albumin solution to remove dead cells. The cells were then washed with 4\% albumin solution and twice with MEM/F-12 (Gibco, Cat. No. 11039) with added $0.2 \%$ albumin and $50 \mu \mathrm{g} / \mathrm{ml}$ gentamycin. Because the culture system of the rapid attachment model does not contain serum, the growth of contaminated non-cardiomyocytes is not problematic. Moreover, adult cardiomyocytes are very highly differentiated and unique in shape, so that they can be easily distinguished from non-cardiomyocytes. Rod-shaped cells were concentrated at $2.5 \times 10^{4}$ cells $/ \mathrm{ml}$. Samples of 2 and $10 \mathrm{ml}$ were plated on to 35- and 100-mm laminin-coated dishes, respectively, or $4 \mathrm{ml}$ in to slide glass chambers. The samples were incubated for $1 \mathrm{~h}$ in a $\mathrm{CO}_{2}$ incubator $\left(95 \%\right.$ air $\left.-5 \% \mathrm{CO}_{2}\right)$ to allow the cells to attach to the bottom, after which they were gently washed 10 times for complete removal of nonattached cells. This was the starting point of the present study (Day 0). The cells were again incubated with MEM/F12 in a $\mathrm{CO}_{2}$ incubator and used for experiments after 0 (Day 0), 1 (Day 1), 2 (Day 2), 3 (Day 3), 5 (Day 5), and 7 days (Day 7). The cardiomyocytes plated on to $35-\mathrm{mm}$ dishes were used for examining morphology and membrane permeability by light microscopy, those in the slide glass chambers were used for electron microscopy and in situ nick end-labeling (TUNEL), and those on the 100-mm dishes were for DNA extraction. Each experiment was done in triplicate.

Non-attached, floating cells were not observed on Day 0. On Day 1 and later, however, floating cells appeared in increasing numbers with repeated washing with culture medium and were also used for experiments. Then, the dishes planned for experiments on later days were supplemented with fresh medium.

\section{Light Microscopy}

The number of attached cells was counted using $1 \mathrm{~mm}^{2}$ grids plated on to the dishes. Non-attached, floating cells were also counted using a hematocounter.

Under a phase-contrast microscope, cells were classified into 2 groups according to the cell length-to-width ratio: rod-shaped cells with a cell length/width ratio greater than 3 and non-rod cells with a cell length/width ratio less than 3 . The latter included square cells and round cells, with or without budding or blebbing, and cellular fragmentation.

Evaluation of Membrane Permeability Cells were exposed to $0.1 \%$ trypan blue for $5 \mathrm{~min}$ and processed for sarcolemmal membrane damage. The number of stained and unstained cells in the dishes was counted and each experiment was done in triplicate in each group. The floating cells were processed in the same way.

\section{Transmission Electron Microscopy}

At Days 0,1,2,3,5, and 7, attached cells and floating cells were separately fixed with phosphate-buffered $2.5 \%$ glutaraldehyde $(\mathrm{pH} 7.4)$ for $4 \mathrm{~h}$ followed by postfixation with $1 \%$ osmium tetroxide for $1 \mathrm{~h}$. They were dehydrated through a graded series of ethanol and embedded in Epon medium. Thin sections were stained with uranyl acetate and lead citrate and examined in a Hitachi H-800 electron microscope.

Quantitative assessment at the electron microscopic level was performed. Cardiomyocytes sectioned at the level of the nucleus were counted and classified as living, apoptotic, and oncotic cardiomyocytes. Apoptotic cardiomyocytes were further classified into cells with intact plasma membranes and those with ruptured plasma membranes. Approximately 200 cardiomyocytes were counted in each group.

\section{DNA In Situ Nick End Labeling (TUNEL)}

The cells cultured on slide chambers were fixed with $1 \%$ paraformaldehyde for $15 \mathrm{~min}$ at room temperature. TUNEL was performed using an ApopTag in situ apoptosis detection kit (Oncor) and then the slides were counterstained with hematoxylin. Floating cells were smeared onto a slide glass, dried and then fixed and stained in the same manner.

\section{DNA Extraction and Electrophoresis}

DNA released from $2.5 \times 10^{5}$ cardiomyocytes (including both attached and non-attached cells) was extracted and separated by electrophoresis in agarose gels according to the method of Arends et al?

\section{Statistical Analysis}

Values were expressed as the mean \pm SEM. Statistical comparisons were performed by ANOVA followed by Newman-Keul's multiple comparisons test. A p value less than 0.05 was considered significant.

\section{Results}

Time Course of the Number of Cardiomyocytes Categorized by Morphology and Membrane Permeability

On Day 0 immediately after removal of non-attached cells, the total number of attached cardiomyocytes was 55 $27 \times 10^{3}$ cells $/ 35-\mathrm{mm}$ dish. The rod-shaped cells averaged $86 \%$ and the dye-excluding cells $98 \%$. During incubation, floating cells appeared and the total number of attached and floating cells decreased rapidly initially and then gradually on the later days (Fig 1A). The reduction in the total cell number was especially extreme during the first day of culture: $32 \%$ reduction. The total cell number finally reached $8.3 \pm 2.1 \times 10^{3}$ cells/dish after 7 days culture ( $88 \%$ reduction). In addition, there were dye-including cells among the 7-day culture cells. Thus, the cell death rate was extreme in the rapid attachment model of cultured cardiomyocytes used in the present study. The number of both rod-shaped and dyeexcluding cells decreased with the incubation period (Fig 1B).

The dye exclusion method may underestimate cell death because apoptotic cell death generally progresses without 
A Cell Number ( $\times 10^{3 / 35-m m ~ d i s h) ~}$

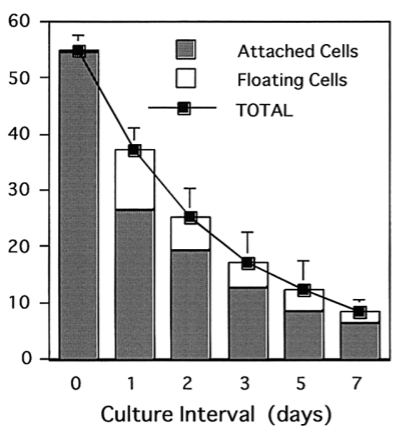

C

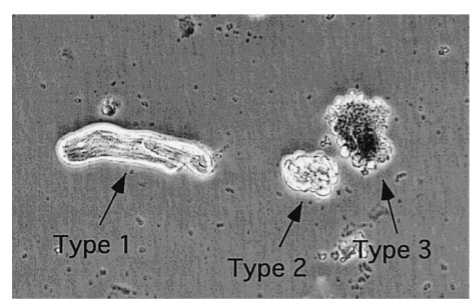

B

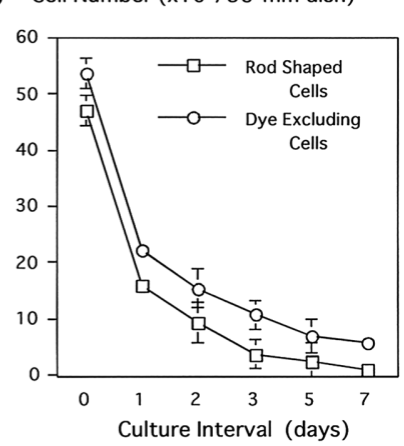

D Cell Number (x103/35-mm dish)

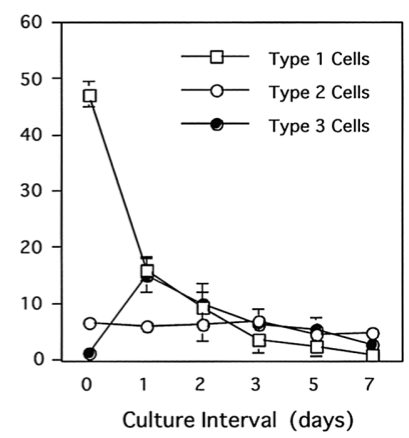

Fig 1. Time course of cardiomyocyte population in 35-mm dishes (A,B,D). (A) Obliquely lined column, number of attached cells; blank column, number of floating cells; solid square, total cell number (a sum of attached and floating cell numbers). The total cell number was significantly decreased as early as 1 day later, compared with the initial value. (B) Blank square plots, rod-shaped cells; blank round plots, dye-excluding cells. The number of dye-excluding cells always surpassed that of rod-shaped cells. In each, the number was significantly decreased at Day 1 and thereafter. (C) Light micrographs of type 1, type 2, and type 3 cardiomyocytes classified by morphology and trypan blue dye staining. (D) Blank square plots, type 1 cells; blank round plots, type 2 cells; solid round plots, type 3 cells. Type 1 cell continued to decrease, type 2 were constant in number and type 3 initially increased and became constant later.

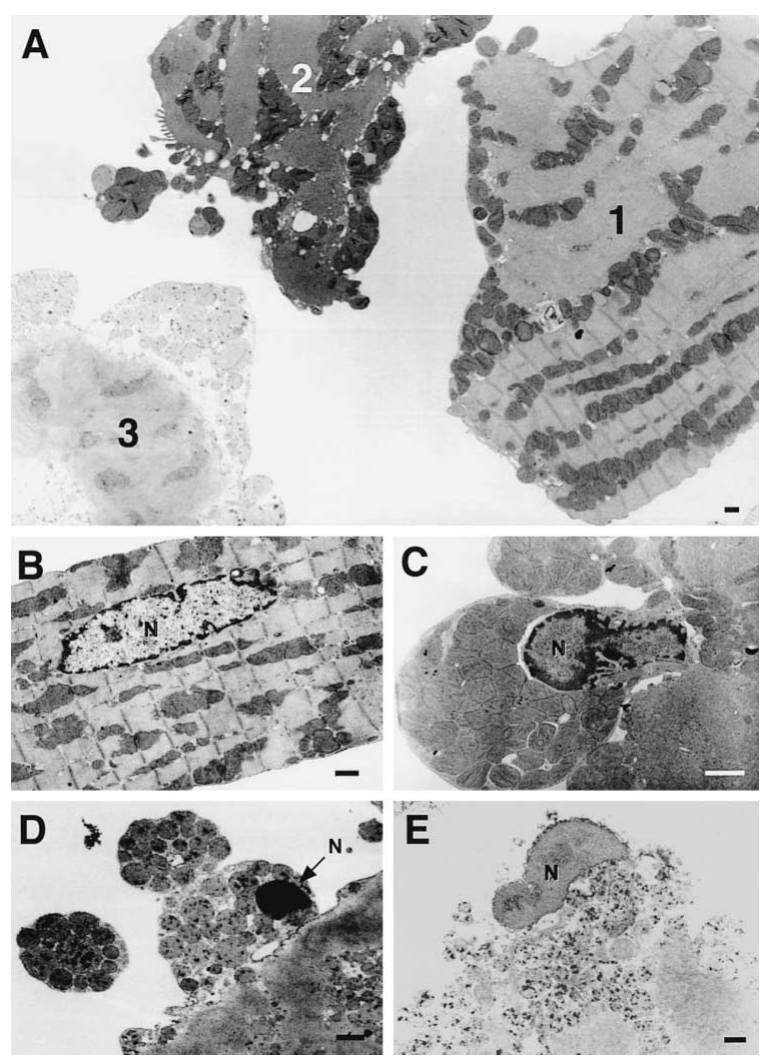

plasma membrane rupture until the later phase ${ }^{1-13}$ In our study the rod-shaped cells were usually less in number than the dye-excluding cells (Fig 1B) and none of the cardiomyocytes showed both a rod shape and dye inclusion. There-
Fig 2. Transmission electron microphotographs of cultured isolated adult cardiomyocytes. (A) Living (1), apoptotic (2), and necrotic (3) cardiomyocytes seen at Day 2. Note the conspicuous difference in the electron density of the cytoplasm among these 3 types of cardiomyocytes: $2>1>3$. (B) A rod-shaped cardiomyocyte with intact membrane, corresponding to type 1 cells at the light microscopic level. Subcellular structure is entirely intact, so this cell is viable and normal. (C) A non-rod-shaped cardiomyocyte with intact membrane (type 2 cell), but showing cytoplasmic shrinkage and characteristic nuclear chromatin condensation with glossy appearance, indicating that this cell is undergoing apoptotic death. (D) Another non-rod-shaped cardiomyocyte with ruptured plasma membrane (type 3 cell) and a subcellular structure showing apoptosis-characteristic features such as electron dense cytoplasm, nuclear chromatin condensation and apoptotic body formation. Thus, this cell is regarded as undergoing postapoptotic necrosis. (E) A non-rod-shaped cardiomyocyte with ruptured plasma membrane, corresponding to a type 3 cells, showing subcellular oncotic changes such as swollen mitochondria with amorphous dense body and non-apoptotic nucleus. Bars $=1 \mu \mathrm{m}$.

fore, the cardiomyocytes were classified into 3 types based on morphology (rod or non-rod) and membrane integrity (dye-exclusion or inclusion): type 1, rod cells with intact membranes; type 2, non-rod cells with intact membranes; 


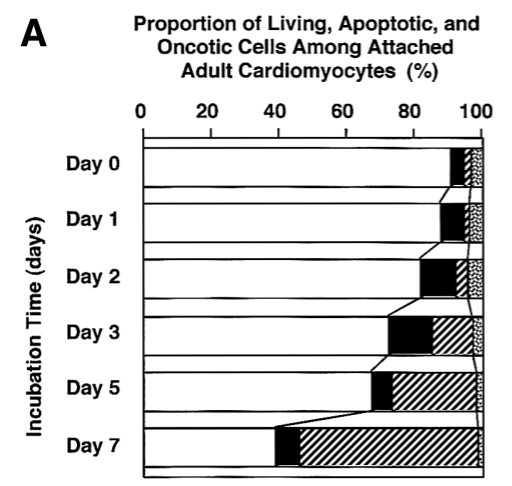

$\square$ Living cells

\section{B}
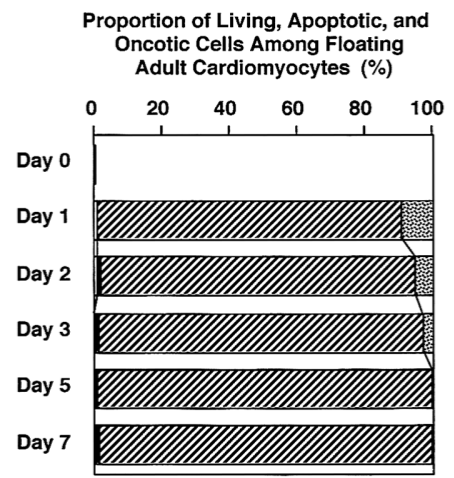

C

Calculated Absolute Numbers of Living, Apoptotic, and Oncotic Cells ( $\times 10^{3}$ cells $/ 35-\mathrm{mm}$ dish)

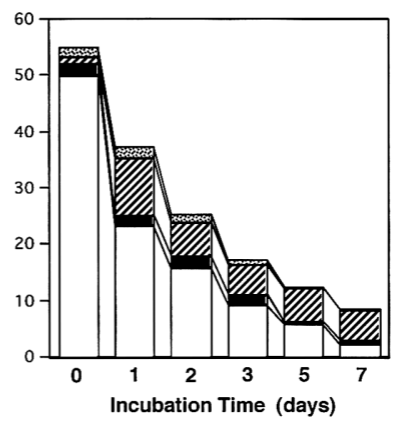

图 Oncosis

Fig 3. Quantitative assessment of cardiomyocytes at the electron microscopic level. The proportions of living, apoptotic, and oncotic cardiomyocytes among the attached cells (A) and among floating cells (B) at Days $0,1,2,3,5$, and 7. (C) The calculated absolute numbers of living, apoptotic, and oncotic cells at Days 0,1,2,3,5, and 7, based on the combination of the findings shown in Figs 1A and 3A,B.

A

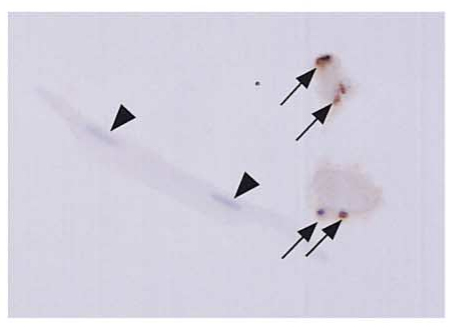

B

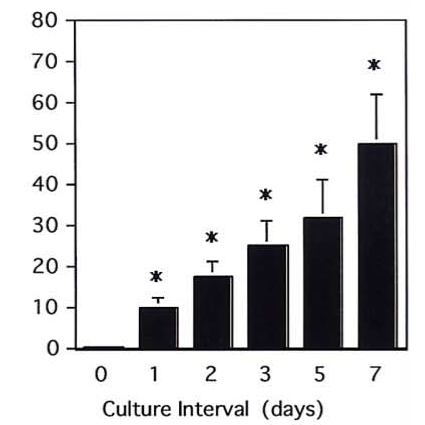

C

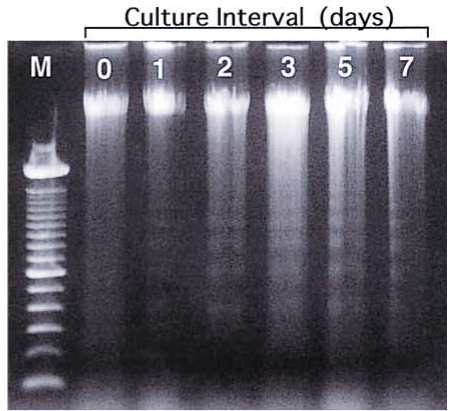

Fig 4. DNA fragmentation in isolated adult rat cardiomyocytes in culture. (A) TUNEL stain of attached cardiomyocytes cultured for 2 days. TUNEL-positive nuclei are indicated by arrows; arrowheads indicate TUNEL-negative nuclei. (B) Incidence of TUNEL-positive cardiomyocytes (apoptotic index) among attached cultured adult cardiomyocytes. ${ }^{*} \mathrm{p}<0.05$ compared with Day 0. (C) DNA gel electrophoresis. A DNA ladder pattern is observed in cardiomyocytes cultured for 2 days (Day 2) and longer. M, 100-bp marker ladder.

and type 3, non-rod cells with ruptured membranes (Fig 1C). During the time course of each cell type, the number of type 1 cells continued to decrease, that of type 2 cells was almost constant and type 3 cells initially increased and then became constant later, at 7 days incubation (Fig 1D).

\section{Ultrastructure of Cardiomyocytes}

To distinguish between the modes of death of cardiomyocytes, we used electron microscopy, which can distinguish between living cells, apoptosis, and oncosis (primary necrosis) (Fig 2). Apoptotic cardiomyocytes were shriveled with relatively intact subcellular organelles and had nuclei with well-defined, glossy chromatin condensation and an intact plasma membrane, all of which was compatible with apoptosis ${ }^{1-13}$ There were cardiomyocytes showing apparently ruptured plasma membranes and degraded mitochondria, but also apoptotic chromatin condensation and cellular fragmentation (ie, apoptotic bodies) and these cells had also died via apoptosis (post-apoptotic secondary necrosis). Oncotic cardiomyocytes were edematous and contained severely degraded subcellular organelles, representative swollen mitochondria containing amorphous dense bodies, and their plasma membranes were ruptured. It was some- times difficult to distinguish between oncosis and apoptosis with ruptured plasma membrane. In such cases, the nuclear morphology (apoptotic or not) was the key for distinction (Fig 2D,E). We also observed cellular debris, which was abundant among the floating cells on Day 1, but decreased on the later days.

Electron microscopy identified the type 1 cardiomyocytes classified by light microscopy as viable, the type 2 as viable or apoptotic and type 3 as oncotic or secondary post-apoptotic necrosis. Fig 3A,B shows the morphometric assessment of attached and floating cardiomyocytes at each incubation time. The analysis revealed that proportionally, oncosis was decreased, but apoptosis was increased. However, since the absolute number of cardiomyocytes continued to decrease with the incubation time, as shown in Fig 1, the result could be interpreted as follows. Oncotic cell death was concentrated during the early phase of culture whereas apoptotic cell death occurred throughout the culture period (Fig 3C).

\section{DNA Fragmentation in Cardiomyocytes}

TUNEL-positivity was not observed in cardiomyocytes at Day 0 , but could be observed among the cardiomyocytes 
on the later days of culture (Fig 4A). A substantial portion of the floating cells were TUNEL-positive, and the incidence of TUNEL-positive cells among the total number of cells increased in parallel with the incubation period (Fig 4B). DNA gel electrophoresis revealed a clear ladder pattern in the cardiomyocytes cultured for 2 days and longer (Fig 4C).

\section{Discussion}

Not only attached but also floating cells were taken into account in the present study and the sum of all cells went on decreasing during culture (Fig 1A). The decrease in the total cell number was mainly caused by the removal of floating cells while changing the medium and partly by cell disappearance through complete degradation after death. In fact, there was abundant cell debris among the floating cells on Day 1. In addition to this cell loss, an overwhelming majority of the floating cells contained dye. Moreover, there were dye-including cells among the attached cells. Taking these findings together, the incidence of dead cells was extremely high. When compared with Day $0,60 \%$ of the cells were dead after 1 day of culture, $81 \%$ after 3 days of culture, and $90 \%$ after 7 days of culture. Because the dye-exclusion method does not detect the early phase of apoptosis that is not accompanied by membrane rupture, the real incidence of death must be higher. Because of this high incidence of spontaneous death, the present information is critically important for the interpretation of studies using this cell type in culture, particularly when cell kinetics are studied.

In the present study, type 3 cells (non-rod and dyeincluding cells) were observed more frequently during the early days of culture than in the later phase, whereas type 2 cells (non-rod and dye-exclusion cells) were almost constantly seen. Cells classified as type 3 at the light microscopic level were oncotic or undergoing post-apoptotic secondary necrosis when observed with electron microscopy, and type 2 cells on light microscopy consisted of viable cells or apoptotic cells at the electron microscopic level. This suggests that oncotic cell death is concentrated during the early phase of culture whereas apoptotic cell death occurs throughout the culture period. Ultrastructural morphometry confirmed this finding and revealed that apoptosis, rather than oncosis, was the predominant mode of spontaneous death of isolated adult cardiomyocytes in culture. In the present model, a DNA ladder appeared and more than $10 \%$ of the cardiomyocytes showed an apoptotic ultrastructure as early as Day 2. This finding should be taken into consideration when the rapid attachment model of adult cardiomyocytes is used for experiments.

The cell loss was unexpectedly extreme during the first day of culture (32\% reduction) and because abundant cell debris was seen among the floating cells of Day 1, complete cell degradation may substantially contribute to this cell loss. However, it was difficult to determine the original mode of death of these lost cells. We additionally examined adult cardiomyocytes that were cultured for $12 \mathrm{~h}$ and found on electron microscopy that the floating cells collected after $12 \mathrm{~h}$ culture had a substantial proportion of oncotic cells $(59 \pm 6 \%, n=3)$. Taking this into account, the rapid cell decrease during the first day was mainly the result of oncotic cell death and subsequent cell degradation. When considering that oncotic cell death mostly occurred during the early phase of culture, it may be that the most important factor triggering oncosis of the cultured cardiomyocytes is injury related to the cell isolation procedure and the subsequent failure in repair.

Majno and Joris proposed, on a morphological basis, 2 modes in the progression of cellular changes towards death as (1) from oncosis to necrosis and (2) from apoptosis to necrosis ${ }^{13}$ Yamamoto et al had previously reported that the mode of spontaneous death of isolated adult rat cardiomyocytes was apoptosis, they used cardiomyocytes from the redifferentiated model, which is a rather exceptional model of adult cardiomyocyte culture. They reported cardiomyocyte apoptosis on the basis of the presence of DNA fragmentation, a biochemical hallmark of apoptosis, 10 but recent studies have shown that DNA fragmentation becomes positive even during the oncotic (necrotic) process ${ }^{15-17}$ The TUNEL procedure positively labels living vascular smooth muscle cells with active RNA transcription ${ }^{18}$ and living cardiomyocytes under DNA repair ${ }^{19}$ thus, evidence of the characteristic ultrastructure, the morphological hallmark of apoptosis ${ }^{11-13}$ is needed for specific detection of apoptosis. Yamamoto et al did not refer to oncotic cardiomyocyte death and because they did not perform an ultrastructural examination, it is not clear whether oncosis occurred in their model. In contrast, the present study demonstrated both apoptotic and oncotic cell death during the natural course of cultured adult rat cardiomyocytes in the rapid attachment model, a popular model in which the short life of the cells is problematic, based on both ultrastructural and biochemical findings.

The possible causes of apoptosis of isolated adult rat cardiomyocytes in culture are lack of growth factors, lack of electrical signals, lack of contractile activity, lack of normal mechanical loads, mild injury related to cell isolation and the early loss of cell contact? In particular, loss of cell contact has recently attracted attention as an important trigger for apoptosis, called anoikis ${ }^{20,21}$ and serum depletion was recently reported to cause apoptosis in cultured neonatal rat cardiomyocytes ${ }^{2}$ Piper's group recommended supplementation of the basic medium with insulin, creatine, carnitine, and taurine to improve the longevity of isolated adult cardiomyocytes in serum-free culture, ${ }^{23}$ and of these, insulin has been recently found to prevent apoptosis of cultured cardiac myocytes 24,25 Thus, the anti-apoptotic effect of the other additives that are considered to improve survival of cultured cardiac myocytes should be investigated.

The major question which arises is why apoptosis has not been previously described in the numerous previous studies of cultured cardiomyocytes. Because many of them have been mainly electron microscopic studies, it is suggested that it was simply overlooked or neglected because the concept of apoptosis was not well established. For example, Vander Heide et al presented an electron microphotograph in which there is an apparent apoptotic body (right lower portion of figure 7 of their report ${ }^{26}$ ), but they did not refer to it as apoptosis. This reveals the immaturity of the research into apoptosis in the field of cardiovascular science and therefore, we suggest that the present findings, although relatively basic, are important and that more information need to be accumulated.

\section{Acknowledgments}

Thanks are due to Akiko Tsujimoto for technical assistance.

This study was supported, in part, by Grants-in-Aid for Scientific Research (Nos 11670668 and 12670704) from the Ministry of Education, Science, Sports and Culture of Japan. 


\section{References}

1. Jacobson SL, Piper HM: Cell cultures of adult cardiomyocytes as models of the myocardium. J Mol Cell Cardiol 1986; 18: 661-678

2. Jacobson SL: Culture of spontaneously contracting myocardial cells from adult rats. Cell Struct Funct 1977; 2: 1-9

3. Claycomb WC, Palazzo MC: Culture of the terminally differentiated adult cardiac muscle cell: A light and scanning electron microscope study. Dev Biol 1980; 80: 466-482

4. Nag AC, Cheng M: Adult mammalian cardiac muscle cells in culture. Tissue Cell 1981; 13: 515-523

5. Piper HM, Probst I, Schwartz P, Hutter JF, Spieckermann PG: Culturing of calcium stable adult cardiac myocytes. $\mathrm{J} \mathrm{Mol} \mathrm{Cell}$ Cardiol 1982; 14: 397-412

6. Framer BB, Mancina M, Williams ES, Watanabe AM: Isolation of calcium tolerant myocytes from adult rat heart: Review of the literature and description of a method. Life Sci 1983; 33: 1-18

7. Ganote CE, Vander Heide RS: Irreversible injury of isolated adult rat myocytes: Osmotic fragility during metabolic inhibition. Am J Pathol 1988; 132: $212-222$

8. Yamamoto S, Yasui K, Palade PT, James TN: Spontaneous death of isolated adult rat cardiocytes in culture in association with internucleosomal cleavage of genomic DNA. Apoptosis 1997; 2: 178-188

9. Wyllie AH: Glucocorticoid-induced thymocyte apoptosis is associated with endogenous endonuclease activation. Nature 1980; 284: $555-556$

10. Arends MJ, Morris RG, Wyllie AH: Apoptosis: The role of the endonuclease. Am J Pathol 1990; 136: 593-608

11. Kerr JFR, Wyllie AH, Currie AR: Apoptosis: A basic biological phenomenon with wide-ranging implications in tissue kinetics. $\mathrm{Br} J$ Cancer 1972; 26: 239-257

12. Buja LM, Eigenbrodt ML, Eigenbrodt EH: Apoptosis and necrosis: Basic types and mechanisms of cell death. Arch Pathol Lab Med 1993; 117: $1208-1214$

13. Majno G, Joris I: Apoptosis, oncosis, and necrosis: An overview of cell death. Am J Pathol 1995; 146: 3-15

14. Khalid MA, Ashraf M: Direct detection of endogenous hydroxyl radical production in cultured adult cardiomyocytes during anoxia and reoxygenation: Is hydroxyl radical really the most damaging radical species? Circ Res 1993; 72: 725-736

15. Collins RJ, Harmon BV, Gobé GC, Kerr JFR: Internucleosomal DNA cleavage should not be the sole criterion for identifying apoptosis. Int J Radiat Biol 1992; 61: 451-453

16. Dong Z, Saikumar P, Weinberg JM, Venkatachalam MA: Internucleosomal DNA cleavage triggered by plasma membrane damage during necrotic cell death. Am J Pathol 1997; 151: 1205-1213

17. Ohno M, Takemura G, Ohno A, Misao J, Hayakawa Y, Minatoguchi $\mathrm{S}$, et al: 'Apoptotic' myocytes in infarct area in rabbit hearts may be oncotic myocytes with DNA fragmentation: Analysis by immunogold electron microscopy combined with in situ nick end-labeling. Circulation 1998; 98: $1422-1430$

18. Kockx MM, Muhring J, Knaapen MWM, de Mayer GRY: RNA synthesis and splicing interferes with DNA in situ end labeling techniques used to detect apoptosis. Am J Pathol 1998; 152: 885-888

19. Kanoh M, Takemura G, Misao J, Hayakawa Y, Aoyama T, Nishigaki $\mathrm{K}$, et al: Significance of myocytes with positive DNA in situ nick end-labeling (TUNEL) in hearts with dilated cardiomyopathy: Not apoptosis but DNA repair. Circulation 1999; 99: 2757-2764

20. Frisch SM, Francis H: Disruption of epithelial cell-matrix connections induces apoptosis. J Cell Biol 1994; 124: 619-626

21. Ding B, Price RL, Goldsmith EC, Borg TK, Yan X, Douglas PS, et al: Left ventricular hypertrophy in ascending aortic stenosis mice: Anoikis and the progression to early failure. Circulation 2000; 101: 2854-2862

22. Wang L, Ma W, Markovich R, Chen JW, Wang PH: Regulation of cardiomyocyte apoptotic signaling by insulin-like growth factor I. Circ Res 1998; 83: 516-522

23. Volz A, Piper HM, Siegmund B, Schwartz P: Longevity of adult ventricular rat heart muscle cells in serum-free primary culture. $J$ Mol Cell Cardiol 1991; 23: 161-173

24. Jonassen AK, Brar BK, Mjos OD, Sack MN, Latchman DS, Yellon DM: Insulin administered at reoxygenation exerts a cardioprotective effect in myocytes by a possible anti-apoptotic mechanism. Circulation 2000; 32: 757-764

25. Aikawa R, Nawano M, Gu Y, Katagiri H, Asano T, Zhu W, et al: Insulin prevents cardiomyocytes from oxidative stress-induced apoptosis through activation of PI3 kinase/Akt. Circulation 2000; 102: $2873-2879$

26. Vander Heide RS, Angelo JP, Altschuld RA, Ganote CE: Energy dependence of contraction band formation in perfused hearts and isolated adult myocytes. Am J Pathol 1986; 125: 55-68 